\title{
Biofilm Formation and Virulence of Shigella flexneri is Modulated by pH of Gastrointestinal Tract
}

I-Ling Chiang ${ }^{1}$, Yi Wang ${ }^{1}$, Satoru Fujii ${ }^{2}$, Brian D. Muegge ${ }^{3}$, Qiuhe Lu ${ }^{2}$, Thaddeus Stappenbeck $^{2} *$

${ }^{1}$ Department of Pathology and Immunology, Washington University School of Medicine, Saint Louis, Missouri 63110, USA.

${ }^{2}$ Department of Inflammation and Immunity, Cleveland Clinic Lerner Research Institute, Cleveland, Ohio 44195, USA.

${ }^{3}$ Division of Endocrinology, Metabolism \& Lipid Research, Department of Medicine, Washington University School of Medicine, St. Louis, Missouri 63110, USA.

*Corresponding author: stappet@ccf.org 


\begin{abstract}
Shigella infections remain a major public health issue in developing countries. One model of Shigella pathogenesis suggests that the microfold epithelial cells in the small intestine are the preferred initial site of infection. However, a growing body of evidence supports an alternative model whereby Shigella primarily infects a much wider range of epithelial cells that reside primarily within the colon. Here, we investigated whether the luminal $\mathrm{pH}$ difference between the small intestine and colon could provide evidence in support of either model of Shigella flexneri pathogenesis. As virulence factors leading to cellular invasion are linked to biofilms in S. flexneri, we examined the effect of $\mathrm{pH}$ on $S$. flexneri's ability to form and maintain adherent biofilms when induced by deoxycholate. We showed that a basic $\mathrm{pH}$ inhibited formation and dispersed pre-assembled mature biofilms while an acidic $\mathrm{pH}$ (similar to the colonic environment) did not have either of these effects. To further elucidate this phenomenon at the molecular level, we probed the transcriptomes of biofilms and S. flexneri grown in different $\mathrm{pH}$ conditions. We identified specific amino acid metabolic pathways (cysteine and arginine) that were enriched in the bacteria that formed the biofilms, but decreased upon $\mathrm{pH}$ increase. We then utilized a type III secretion system reporter strain to show that increasing $\mathrm{pH}$ reduced deoxycholate-induced virulence of $S$. flexneri in a dose dependent manner. Taken together, these experiments support a model whereby Shigella infection is favored in the colon because of the local $\mathrm{pH}$ differences in these organs.
\end{abstract}




\section{Introduction}

Shigella spp. are gram-negative bacterial pathogens that cause approximately $5-15 \%$ of the diarrheal burden in the world $(1,2)$. Infection can have serious consequences as Shigella infection results in $\sim 200,000$ deaths per year, mostly in pediatric populations of developing countries (2). Shigella is most efficiently transmitted human to human by a fecal-oral route and can invade intestinal epithelial cells $(3,4)$. Despite the morbidity and mortality resulting from Shigella infections, clinically termed shigellosis, there is not an effective vaccine for the bacteria nor do we fully understand the mechanism by which this pathogen infects the gastrointestinal $\operatorname{tract}(5)$.

One major limitation that has slowed our understanding of Shigella infections is that humans are the main reservoir for this microbe. Primates can be infected, but establishment of other in vivo animal infection models that recapitulate the phenotypes of enteric invasion has been challenging (6). Data from infection of ex vivo ligated rabbit ileal loops suggests that Shigella are phagocytosed by specialized epithelial microfold ("M") cells that are most prominent overlying organized gut-associated lymphoid tissue (GALT). These M cells facilitate bacterial translocation to immune cells that underlie their basolateral surface (7). One shortcoming of this model is that in humans M cells are primarily found in ileal Peyer's patches of the small intestine, whereas Shigella infection occurs predominantly in the colon $(1,8)$. Physiological and pathological studies of Shigella infections in humans and rhesus monkeys, respectively, demonstrate that the bacteria primarily invade and damage the colonic epithelium in areas not necessarily associated with GALT and has minimal effects on the small intestine (9, 10). However, in vitro primary gut epithelium infection studies showed that Shigella can successfully invade epithelial cells derived from both small and large intestines (11). Based on the discrepancies between in vivo and in vitro observations, we hypothesize that differences between the luminal environments of small intestine and colon account for the preferential tropism of Shigella for the colon.

Bile is one important luminal factor that enteric pathogens must navigate in order to colonize and invade into the intestinal wall. It is a heterogeneous mixture of primary and secondary bile salts, cholesterol, phospholipids, and bilirubin that facilitates the digestion of fat in the small intestine (12). Pathogenic bacteria, including Escherichia coli, Vibrio cholerae, Campylobacter jejuni and Shigella, have evolved mechanisms to resist the antimicrobial 
properties of bile or even utilize bile as a signal to regulate virulence (13). Relevant here, upon exposure to bile salts, Shigella increases secretion of virulence factors, thus increasing adhesion to and invasion of host cells $(14,15)$. Additionally, long-term bile salt exposure in vitro induces Shigella biofilm formation, a phenomenon also observed when $V$. cholerae encounters bile salts $(15,16)$. A large percentage of bile salts are absorbed in the ileum, and approximately 400-800 mg of bile salts enter the colon daily (17). Thus, despite its importance in increasing pathogenic potency, it is unlikely that bile salts are the sole factor explaining the preferential colonic invasions by Shigella.

Concurrent with the inflow of bile into the small intestine, however, is the influx of bicarbonate $\left(\mathrm{HCO}_{3}{ }^{-}\right)$, the physiological base used to buffer acidic contents from the stomach which creates a basic luminal environment in the distal small intestine (mean of 7.7, range for most subjects is $\mathrm{pH} 7.4-8)(18,19)$. In contrast, the colonic lumen supports a more acidic environment (average $\mathrm{pH}$ is 6.4 though there is high variation, range of $\mathrm{pH} 5-8)(18,19)$. Interestingly, bicarbonate modulates virulence factors, toxin production, and biofilm formation in $V$. cholera $(16,20,21)$. However, there is a paucity of data regarding interactions between bicarbonate and Shigella. Given the drastic $\mathrm{pH}$ alteration from ileum to colon and the role of $\mathrm{pH}$ in regulating pathogenic activity of Vibrio Cholerae, we hypothesized that $\mathrm{pH}$ plays a vital role in regulating bile salts-dependent virulence of Shigella and its preferential pathogenesis in the colon.

In this study, we utilized previously established biofilm formation methods to test whether deoxycholate-induced biofilms of Shigella flexneri could form under various $\mathrm{pH}$ conditions. We demonstrated that more basic $\mathrm{pH}$ levels, as observed in the small intestine, attenuated biofilm formation without causing bacterial cell death. Additionally, we showed that basic $\mathrm{pH}$ conditions dispersed mature biofilms. RNA sequencing of $S$. flexneri under various $\mathrm{pH}$ conditions showed differences in transcriptional profiles of bacteria grown in deoxycholate with or without $\mathrm{NaOH}$. Using a type III secretion system reporter strain of Shigella, we demonstrated that increasing $\mathrm{pH}$ could downregulate virulence as well. Collectively, these studies demonstrate that basic conditions, as found in the lumen of the small intestine, are not favorable for $S$. flexneri pathogenesis and provide new mechanistic insight into shigellosis pathogenesis. 


\section{Results}

\section{Basic Conditions Attenuate Deoxycholate-induced Biofilm Formation}

Deoxycholate is a secondary bile salt that is present throughout both small and large intestines and is known to enhance secretion of S. flexneri virulence factors in planktonic culture (14). We initially tested if this bile acid affected the ability of S. flexneri to form biofilms in vitro (Figure 1A). To optimize conditions to produce $S$. flexneri biofilms, we grew $S$. flexneri in tryptic soy broth (TSB) supplemented with varying concentrations of sodium deoxycholate (NaDCA) and then measured the opacity of biofilms by optical density at $600 \mathrm{~nm}\left(\mathrm{OD}_{600}\right)$. Incubation of $S$. flexneri with $0.05 \% \mathrm{NaDCA}$ stimulated formation of biofilms that approached $50 \%$ of the maximal opacity, and was thus suitable for testing the effects of external factors on biofilm formation (Figure 1B).

We hypothesized that alterations of extracellular $\mathrm{pH}$ would impact biofilm formation, as the $\mathrm{pH}$ of the intestinal lumen is known to vary along its length (18). To simulate the basic conditions found in the small intestine, we adjusted the $\mathrm{pH}$ of the TSB media containing $0.05 \%$ $\mathrm{NaDCA}$ with increased concentrations of $\mathrm{NaOH}$. We characterized the resulting biofilms by $\mathrm{OD}_{600}$ to measure opacity, MTT assay to assess microbial viability, and colony forming units to quantify the number of planktonic bacteria in the media that were not incorporated into the biofilm (Figure 1C, 2A and 2B). Increasing basic concentration in the TSB media decreased the opacity of the biofilms (Figure 2C) in a dose dependent manner with an almost complete loss of biofilm at $20 \mathrm{mM} \mathrm{NaOH}$ (Figure 2E). Importantly, the $\mathrm{pH}$ that corresponds to 5 and $10 \mathrm{mM}$ is in the range of most human subjects in the distal small intestine (19). MTT assays, which have been utilized by many other biofilm studies, corroborated the reduction in biofilm formation with $\mathrm{NaOH}$ treatment (Figure 2D). Importantly, the attenuation of biofilm formation by increased $\mathrm{pH}$ did not appear to be secondary to enhanced bacterial killing by $\mathrm{NaOH}$. In fact, the planktonic phase of $S$. flexneri that was maintained apically of the biofilm was enhanced by increased $\mathrm{pH}$ as measured by OD600 and CFU/mL (Figure 2F and S1A). Our interpretation is that there was an increased influx of bacteria into the planktonic phase as the biofilm was disassembled by basic conditions.

Taken together, these data support the hypothesis that a basic environmental $\mathrm{pH}$ attenuates NaDCA-induced biofilm formation in S. flexneri, and this effect occurs at a range of $\mathrm{pHs}$ that is similar to the small intestinal lumen (Figure 2B). To further test that basic 
environments relevant to the small intestine can play a role in decreasing deoxycholate-induced biofilm formation, we repeated our assay with $\mathrm{NaHCO}_{3}$, the physiological agent responsible for modulating $\mathrm{pH}$ in the small intestine, and confirmed that a physiologically relevant bicarbonate concentration ( $0.3 \%$ ) (22) could prevent biofilm formation as well (Figure 2G and $\mathbf{2 H}$ ). To control for the possible effects induced by changes in ionic strength, we tested $\mathrm{NaCl}$ of the same concentrations and confirmed that $\mathrm{NaCl}$ did not affect biofilm formation (Figure 2I).

\section{Acidic Conditions Do Not Affect Biofilm Formation, but Decreases Viability of S. flexneri}

To test if the acidic $\mathrm{pH}$ that $S$. flexneri encounters in the large intestine can influence biofilm formation, we supplemented TSB media with increasing concentrations of $\mathrm{HCl}$ (Figure S1B). In contrast to the increased biofilm density observed in basic conditions, we found that acidic pH did not significantly change the opacity of the biofilms (Figure S1C). Despite the presence of biofilms in all acidic conditions, the MTT assay showed diminished activity indicating that either the metabolism or number of bacteria surviving in the biofilms was decreased as the concentration of $\mathrm{HCl}$ was increased (Figure S1D). The number of planktonic bacteria growing in the media above the biofilm was also decreased with $\mathrm{HCl}$ addition as measured by CFU/mL (Figure S1E and S1F). Taken together, we showed that there was no reduction in biofilm assembly and formation in acidic conditions, despite decreased viability and/or metabolism for S. flexneri. These results support a model that $S$. flexneri biofilms are better supported in the colonic environment where there is an acidic milieu that does not prevent bile salt-induced biofilm formation.

\section{Environmental pH Modulates Dispersal of Mature S. flexneri Biofilms}

The preceding experiments established that basic conditions prevent the assembly of biofilms from planktonic cultures. We next tested whether basic conditions affect biofilms that are already assembled and matured. First, we induced biofilm formation with NaDCA and allowed the biofilm to mature for $24 \mathrm{~h}$, and then exchanged the growth media to TSB without $\mathrm{NaDCA}$ at a range of $\mathrm{NaOH}$ concentrations (Figure 3A). We found that the addition of 2.5, 5, or $10 \mathrm{mM} \mathrm{NaOH}$ did not significantly affect the biofilm opacity, though there was a trend towards reduction. A concentration of $20 \mathrm{mM} \mathrm{NaOH}$ treatment fully dispersed the mature biofilm as indicated by decreased opacity (Figure 3B). The effect of basic pHs on biofilm dispersal was not 
a potent as its effect on formation. These results were further supported by MTT assay (Figure 3C). These data suggest that the disruptive effects of $\mathrm{NaOH}$ on $S$. flexneri biofilms was not caused by its deprotonation effect on deoxycholate, but rather by its direct influences on the bacteria. We found that acidic environments did not significantly disperse mature biofilms or reduce bacterial viability (Figure $\mathbf{S 2 A}$ and $\mathbf{S 2 B}$ ).

\section{RNA-Sequencing Reveals that Basic Conditions Attenuate a Biofilm-Associated Transcriptomic Program.}

To gain additional insight into how $\mathrm{pH}$ affects $S$. flexneri biofilm formation, we performed RNA-sequencing to determine the transcriptomic alterations in S. flexneri caused by $\mathrm{pH}$ modulation. We performed this analysis using three experimental conditions that included (a) planktonic cultures in TSB only, (b) biofilms in TSB $+0.05 \% \mathrm{NaDCA}$, and (c) planktonic cultures in TSB $+0.05 \% \mathrm{NaDCA}+20 \mathrm{mM} \mathrm{NaOH}$. A global view of the transcriptional profiles from these experimental groups, as shown by PCA plot, indicated that there were indeed transcriptional differences among each experimental group (Figure S3A).

To identify gene signatures that were associated with biofilm formation, we first compared the mRNA transcriptomic profiles between the deoxycholate-induced biofilm and planktonic S. flexneri grown in TSB only. Pathway analysis (DAVID 6.7) of genes upregulated in the biofilm showed an enrichment of ABC transporters, arginine metabolism, and sulfur metabolism (Figure 4A). Interestingly, arginine and cysteine metabolic pathways have been reported to be important in biofilm formation in other bacterial species $(23,24)$. We next compared the biofilm cultures grown with and without $20 \mathrm{mM} \mathrm{NaOH}$. Pathway analysis revealed that the deoxycholate-induced biofilm gene enrichment of $\mathrm{ABC}$ transporters, arginine metabolism, and sulfur metabolism was repressed by the addition of $\mathrm{NaOH}$ (Figure 4B). Heatmap analysis of representative genes from these pathways further supported the specific increase in these genes only in the NaDCA conditions (Figure 4C). qPCR analysis on candidate genes that are involved in arginine and cysteine metabolism confirmed the RNA-seq results (Figure S3B). Taken together, these data demonstrated that basic conditions suppress a biofilmassociated gene program induced by deoxycholate in S. flexneri. 


\section{Basic Conditions Prevent Induction of T3SS by Deoxycholate}

In addition to stimulating biofilm formation, deoxycholate increases the expression of virulence factors in S. flexneri, especially those related to the type III secretion system (T3SS), the molecular machinery $S$. flexneri utilizes to invade into host cells (1). Given the attenuation of deoxycholate-induced biofilm formation in basic conditions, we hypothesized that addition of $\mathrm{NaOH}$ could also reduce virulence of $S$. flexneri. To test this, we utilized a previously constructed Shigella strain that contains a fluorescent reporter for the T3SS (Shigella M90T Sm pTSAR 2.4) (25). In this system, mCherry is constitutively expressed in all bacteria but GFP is expressed only when the T3SS apparatus is induced. We first demonstrated that addition of $0.2 \%$ NaDCA in TSB induced GFP production in this Shigella reporter strain, whereas bacteria growing in TSB alone did not fluoresce green. When the $\mathrm{pH}$ of the TSB was adjusted with increasing concentrations of $\mathrm{NaOH}$, the T3SS activation by deoxycholate was reduced in a dosedependent manner with 5 and $10 \mathrm{mM}$ concentrations of $\mathrm{NaOH}$ showing significant effects (Figure 5A and 5B). This experiment further supports the model that basic conditions deter Shigella from infection in the small intestine.

\section{Discussion}

In this study, we utilized a series of in vitro bacterial assays, RNA-sequencing, and fluorescent microscopy to demonstrate that a decrease in deoxycholate-regulated biofilm formation and virulence induction of $S$. flexneri occurs under basic $\mathrm{pH}$ conditions. The results of these experiments support a model that $S$. flexneri would preferentially infect the colon over the distal small intestine. We propose that as S. flexneri transits through the distal small intestine, the basic $\mathrm{pH}$ of the luminal environment would act to reduce biofilm formation, virulence, and epithelial invasion. As it reaches the more acidic colonic lumen, the bacteria could increase biofilm formation and virulence-related gene expression which would facilitate epithelial invasion at this site.

$V$. cholera, a pathogen that preferentially colonizes the small intestine, serves as an important comparator to Shigella. In both bacteria, bile salts can induce biofilm formation (16). However, bicarbonate upregulates virulence factors and toxin production in $V$. cholera, whereas the same condition reduces virulence in Shigella $(13,15)$. Thus, our study of Shigella and 
previous studies of $V$. cholera highlight the importance of considering environmental factors in the lumen when studying pathogenesis of specific enteric pathogens that infect different regions of the gut.

Recent advances in the detection of intestinal pathogens using qPCR revealed that Shigella may be twice as prevalent as previously estimated (26). Due to the lack of available vaccines for shigellosis, antibiotics are currently the first line of treatment for this diarrheal disorder (27). However, antibiotic resistance has become an increasingly severe problem in part exacerbated by the excessive antibiotic use. Thus, we urgently need to find alternative approaches to treat bacterial diseases like shigellosis (28). In this study, we elucidate a pHdependent mechanism by which the small intestine evades Shigella invasion. With further investigations on specific pathways that mediate the reduction of virulence and biofilm formation under basic $\mathrm{pH}$ conditions, we can potentially find targets to therapeutically treat shigellosis.

Bacterial biofilms play a clear role in physiology and pathophysiology in many different biologic systems. For example, Vibrio fischeri produces biofilms in the light organ of the Hawaiian squid, Pseudomonas aeuginosa produces biofilms in the lung airways that that can lead to diminished respiratory function and polymicrobial biofilms on the enamel surface of teeth. $(23,24,29)$. Despite the extensive number of studies on bacterial biofilms, there is been minimal characterization of deoxycholate-induced Shigella biofilms. Through RNA-sequencing of Shigella biofilms, we showed that the arginine and cysteine metabolism pathways were upregulated and enriched. The arginine and cysteine metabolism pathways are also associated with $P$. aeruginosa biofilms and $V$. fischeri biofilms, respectively $(30,31)$. Because biofilms in the gut have been potentially associated with colorectal cancer, inflammatory bowel disease, and various enteric infections, our studies may help further the investigation of biofilms related to disorders of the gastrointestinal tract $(32,33)$.

Although more work is needed to further elucidate the pathways and host factors that control Shigella virulence and biofilm formation, our work expands on our knowledge of Shigella pathogenesis and provides a unique perspective into studying mechanisms of infection. This will prove to be imperative as we are slowly attempting to uncover more and more interactions between the host and pathogen. 


\section{Materials and Methods}

\section{Biofilm Formation Assays}

Shigella flexneri strain 2a 2457 T was purchased from ATCC (\#700930) and used within 6 months of receipt. S. flexneri was inoculated into LB media from the original commercial stock and grown $20 \mathrm{~h}$ at $37^{\circ} \mathrm{C}$ with shaking at $250 \mathrm{rpm}$. After this incubation, the bacteria were pelleted by centrifugation, resuspended in tryptic soy broth (TSB), and measured by $\mathrm{OD}_{600}$. The bacterial suspension was then diluted with TSB and adjusted to an $\mathrm{OD}_{600}$ of 0.5 . From this stock, the bacteria were further diluted 1:800 in TSB that included 0.05\% NaDCA. The bacterial suspension was then added to a 96 -well plate (180 $\mu \mathrm{L} /$ well). For experiments $20 \mu \mathrm{L}$ of $\mathrm{H}_{2} \mathrm{O}$, diluted $\mathrm{HCl}$, or diluted $\mathrm{NaOH}$ was added to each well to achieve desired final concentrations. The plate was incubated stationary at $37^{\circ} \mathrm{C}$ for $24 \mathrm{~h}$ to allow for biofilm formation. The supernatant above the resultant biofilm was removed and set aside for $\mathrm{OD}_{600}$ or $\mathrm{CFU} / \mathrm{mL}$ measurement; the biofilms were washed with $100 \mu \mathrm{L} \mathrm{H}_{2} \mathrm{O}$ twice and measured by $\mathrm{OD}_{600}$ before the MTT solution $(100 \mu \mathrm{L} ; 0.5 \mathrm{mg} / \mathrm{mL}$ in TSB) was added to the biofilms. The plate was incubated at $37^{\circ} \mathrm{C}$ for 10 minutes before the MTT solution was removed and dissolved with 100 $\mu \mathrm{L}$ DMSO. The $\mathrm{OD}_{570}$ of the resulting solution was measured by Cytation 5. The planktonic cells in the supernatant were sequentially diluted in PBS and plated onto LB plates for $\mathrm{CFU} / \mathrm{mL}$ quantification. A similar procedure was utilized for the testing of $\mathrm{NaHCO}_{3}, \mathrm{NaCl}$, and the dose curve of NaDCA.

\section{S. flexneri Growth Curve}

S. flexneri was grown overnight in LB media. The bacteria were collected with centrifugation, adjusted to $\mathrm{OD}_{600}$ of 0.5 , and diluted 1:20 in TSB. The bacterial suspension was added to 96-well plates $(180 \mu \mathrm{L} /$ well $)$ and then additional $\mathrm{H}_{2} \mathrm{O}, \mathrm{HCl}$, or $\mathrm{NaOH}$ solutions were added to achieve final volume of $200 \mu \mathrm{L}$ and desired concentrations. The plate was incubated at $37^{\circ} \mathrm{C}$ with shaking, and the $\mathrm{OD}_{600}$ of the plate was measured at 2, 4 and $6 \mathrm{~h}$.

\section{Biofilm Dispersion Assays}

An overnight culture of $S$. flexneri in LB was adjusted to $\mathrm{OD}_{600}$ of 0.5 and further diluted 1:800 in TSB with $0.05 \%$ NaDCA. The diluted bacterial suspension was added to 96-well plates (200 $\mu \mathrm{L} /$ well) and incubated without shaking at $37^{\circ} \mathrm{C}$ for $24 \mathrm{~h}$. After $24 \mathrm{~h}$, the supernatant above the 
biofilm was removed, the biofilms were washed once with $\mathrm{H}_{2} \mathrm{O}(100 \mu \mathrm{L})$ and a new solution of TSB with various concentrations of $\mathrm{NaOH}$ or $\mathrm{HCl}(200 \mu \mathrm{L} /$ well $)$ was added on top of the biofilms. After incubation at $37^{\circ} \mathrm{C}$ with no shaking for $24 \mathrm{~h}$, the resulting biofilms were measured by $\mathrm{OD}_{600}$ and the MTT assay as described above.

\section{RNA Sequencing of S. flexneri}

The diluted solution of bacteria from an overnight culture (first adjusted to $\mathrm{OD}_{600}$ of 0.5 , then 1:20 dilution in TSB) was further diluted to 1:800 in TSB +/- 0.05\% NaDCA. For TSB only control samples, an aliquot of $200 \mathrm{uL} /$ well of the above dilutions (without NaDCA) was added to into 96 well plates. To assess the effects of $\mathrm{NaDCA}$ and/or $\mathrm{NaOH}$, an aliquot of $180 \mu \mathrm{L} / \mathrm{well}$ of the above dilutions (with NaDCA) was added with $20 \mu \mathrm{L}$ of either $\mathrm{H}_{2} \mathrm{O}$ or $200 \mathrm{mM} \mathrm{NaOH}$. The plates were incubated stationary at $37^{\circ} \mathrm{C}$ for $24 \mathrm{~h}$. The planktonic bacteria in the wells containing TSB only (no NaDCA) or TSB with $0.05 \% \mathrm{NaDCA}$ and $20 \mathrm{mM} \mathrm{NaOH}$ were collected and pelleted with centrifugation (15 min, 4C) and lysed according to instructions of RiboPure RNA Purification kit for bacteria (Invitrogen). Biofilms from wells containing TSB and $0.05 \%$ NaDCA were washed and directly lysed with the same kit. RNA was then purified using the RNeasy Mini Kit (Qiagen). Genomic DNA removal was performed according to instructions of TURBO DNA-free Kit (Invitrogen).

Libraries were prepared according the manufacture protocol and sequenced with Illumnia HiSeq3000. The Ensembl release 76 top-level assembly STAR version 2.0.4b was used to align RNA-seq reads and the Subread:feature Count version 1.4.5 was used to derive gene counts. Gene counts were then imported into and analyzed with R/Bioconductor package Edge ${ }^{5}$ for normalization and then imported into R/Bioconductor package Limma. Differential expression was determined with Benjamini-Hochberg false-discovery rate adjusted p-values less than or equal to 0.05. PCA plot was made with DDSeq2 package based on all gene counts. Pathway analysis was performed by utilizing the Database for Annotation, Visualization and Integrated Discovery (DAVID) v6.7 (https://david-d.ncifcrf.gov ). 
For qPCR verification, 500ng of RNA was converted to cDNA using iScript Reverse Transcription Supermix Kit (Bio-Rad) and qPCR was performed with TB Green Advantage qPCR Premix. RecA was used as a housekeeping gene. Primers utilized are shown in Table S1.

\section{Fluorescence Microscopy to Probe Activation of T3SS}

A Congo red positive colony of Shigella M90T Sm transformed with the pTSAR 2.4 plasmid (a kind gift from Dr. François-Xavier Campbell-Valois) was grown in TSB overnight. The strain was transformed following protocol as previously reported (25). The overnight culture was diluted 1:100 into TSB solutions with or without $0.2 \%$ NaDCA under various $\mathrm{pH}$ conditions (adjusted with $\mathrm{H}_{2} \mathrm{O}, \mathrm{HCl}$, or $\mathrm{NaOH}$ ), and incubated for $3 \mathrm{~h}$ without shaking at $37^{\circ} \mathrm{C}$. The activation of type III secretion system was assessed with the GFP channel by fluorescent microscopy (25). 


\section{Achknowledgements}

We thank Dr. François-Xavier Campbell-Valois for providing Shigella M90T Sm and pTSAR

2.4 plasmid. We also thank Shanshan Xiong for her help in this project. I.L.C is supported by a fellowship for MA/MD program from Washington University School of Medicine. Crohn's \& Colitis Foundation provided support. 


\section{Figure Legends}

\section{Figure 1: Experimental procedure utilized in this study}

(A) Diagram showing the two resultant phases of bacterial growth after incubation in TSB with NaDCA overnight at $37^{\circ} \mathrm{C}$.

(B) The opacity of Shigella biofilms induced by increased percentages of sodium deoxycholate (NaDCA) for $24 \mathrm{~h}$ at $37^{\circ} \mathrm{C}$. Note that $0.05 \% \mathrm{NaDCA}$ induced biofilms that were approximately $50 \%$ of the maximal opacity and were selected for subsequent experiments (arrowhead).

(C) Schematic of Shigella biofilm assays grown in $0.05 \%$ NaDCA and exposed to varying $\mathrm{pH}$ conditions. Biofilms were assayed for biofilm density (OD600 biofilm), viability (MTT), planktonic density (OD600 planktonic), and bacteria count (CFU/mL).

NaDCA; sodium deoxycholate. TSB; tryptic soy broth.

Figure 2: The effects of increased $\mathrm{pH}$ on formation of deoxycholate-induced biofilms

(A) Diagram describing the experimental steps for testing the effects of $\mathrm{NaOH}$ on biofilm formation induced by NaDCA.

(B) A table showing the corresponding $\mathrm{pH}$ values when increased concentrations of $\mathrm{NaOH}$ were added to the TSB media with $0.05 \%$ NaDCA.

(C-D) The opacity (as measured by $\mathrm{OD}_{600}, \mathrm{C}$ ) and viability (as measured by MTT assay, D) of biofilms induced by $0.05 \% \mathrm{NaDCA}$ with increased concentrations of $\mathrm{NaOH}$ after $24 \mathrm{~h}$ at $37^{\circ} \mathrm{C}$.

(E) Representative images of biofilms induced by $\mathrm{NaDCA}$ with or without $20 \mathrm{mM} \mathrm{NaOH}$.

(F) Growth curve of the planktonic Shigella (measured by $\mathrm{OD}_{600}$ ) growing on top of the

NaDCA-induced biofilms with increased concentrations of $\mathrm{NaOH}$.

(G-H) Biofilm viability $(\mathrm{G})$ and planktonic growth curve $(\mathrm{H})$ with or without $0.3 \% \mathrm{NaHCO}_{3}$.

(I) Biofilm viability with or without $20 \mathrm{mM} \mathrm{NaCl}$ (as a control for ionic strength).

$\mathrm{n}=3$ independent experiments. All bar graphs were plotted as Mean \pm SD. Statistical significance was determined by Student's t-tests with $\mathrm{p}<0.05$. $* *=$ significant with two-tailed Student's t-test.

\section{Figure 3: Dispersal of biofilms modulated by increased $\mathrm{pH}$}

(A) Illustration on the experimental procedures for testing the effects of $\mathrm{NaOH}$ on biofilm dispersion and disassembly. 
(B-C) The opacity (B) and viability (C) of pre-formed biofilms incubated with increased concentrations of $\mathrm{NaOH}$ for $24 \mathrm{~h}$.

$\mathrm{n}=3$ independent experiments. All bar graphs were plotted as Mean \pm SD. Statistical significance was determined by Student's t-tests with $\mathrm{p}<0.05$. $* *=$ significant with two-tailed Student's t-test.

Figure 4: Transcriptomic analysis of the effects of increased pH on deoxycholate-induced biofilms

(A) Top upregulated pathways from Shigella cultured in TSB with $0.05 \%$ NaDCA compared to TSB only.

(B) Top downregulated pathways from Shigella cultured in TSB with $0.05 \% \mathrm{NaDCA}+\mathrm{NaOH}$ compared to TSB with $0.05 \%$ NaDCA.

(C) Heatmap showing the relative levels of genes involved in the arginine, leucine and cysteine metabolism pathways. Plots represented 2 independent experiments.

\section{Figure 5: Increased pH inhibits deoxycholate-induced virulence of Shigella}

(A) Images showing the effect of sodium deoxycholate and $\mathrm{NaOH}$ on a fluorescent reporter of Shigella flexneri which expresses GFP upon activation of the T3SS (with constitutive expression of mCherry as an internal control). Scale bar $=100 \mu \mathrm{m}$.

(B) Percentage of GFP+ cells out of mCherry+ cells under increased $\mathrm{NaOH}$ concentrations was plotted as Mean \pm SD. $n=2$ independent experiments. Statistical significance was determined by Student's t-tests. **p<0.01 with two-tailed Student's t-test. 


\section{Supplemental Figure Legends}

\section{Figure S1: The effect of decreased pH on Shigella biofilm formation}

(A) CFU/mL measurement of the planktonic Shigella growing on top of NaDCA-induced biofilms with increased concentrations of $\mathrm{NaOH}$ after $24 \mathrm{~h}$ at $37^{\circ} \mathrm{C}$.

(B) A table showing the corresponding $\mathrm{pH}$ values when increased concentrations of $\mathrm{HCl}$ were added to the TSB media with $0.05 \%$ NaDCA.

(C-D) The opacity (C) and viability (D) of biofilms induced by $0.05 \%$ NaDCA with increased concentrations of $\mathrm{HCl}$.

(E) CFU/mL measurement of the planktonic Shigella growing on top of NaDCA-induced biofilms with increased concentrations of $\mathrm{HCl}$ after $24 \mathrm{~h}$ at $37^{\circ} \mathrm{C}$.

(F) Growth curve of the planktonic Shigella (measured by $\mathrm{OD}_{600}$ ) growing on top of the $\mathrm{NaDCA}$-induced biofilms with increased concentrations of $\mathrm{HCl}$ at indicated time points. $\mathrm{n}=3$ independent experiments. All bar graphs were plotted as Mean \pm SD. Statistical significance was determined by Student's t-tests with $\mathrm{p}<0.05$. $* *=$ significant with two-tailed Student's t-test. *=significant with one-tailed Student's t-test. NS=not significant.

\section{Figure S2: Dispersal of biofilms modulated by decreased pH}

(A-B) The opacity (A) and viability (B) of pre-formed biofilms incubated with increased concentrations of $\mathrm{HCl}$ for $24 \mathrm{~h}$.

$\mathrm{n}=3$ independent experiments. All bar graphs were plotted as Mean \pm SD. Statistical significance was determined by Student's t-tests with $\mathrm{p}<0.05$. NS=not significant.

\section{Figure S3: Verification of RNA-sequencing results with qPCR}

(A) PCA plot of the transcriptomes from Shigella cultured in TSB only, TSB with $0.05 \%$ $\mathrm{NaDCA}$ and TSB with $0.05 \% \mathrm{NaDCA}+20 \mathrm{mM} \mathrm{NaOH}$.

(B) qPCR results of various genes involved in arginine and cysteine metabolism were plotted as Mean \pm SD for 2 independent experiments. $n=3$ technical replicates for each experiments. Statistical significance was determined by Student's t-tests. ${ }^{*} \mathrm{p}<0.01$ with two-tailed Student's t-test. $* \mathrm{p}<0.05$ with two-tailed Student's t-test. 


\section{References}

1. Schroeder GN, Hilbi H. 2008. Molecular pathogenesis of Shigella spp.: controlling host cell signaling, invasion, and death by type III secretion. Clin Microbiol Rev 21:134-56.

2. Khalil IA, Troeger C, Blacker BF, Rao PC, Brown A, Atherly DE, Brewer TG, Engmann CM, Houpt ER, Kang G, Kotloff KL, Levine MM, Luby SP, MacLennan CA, Pan WK, Pavlinac PB, Platts-Mills JA, Qadri F, Riddle MS, Ryan ET, Shoultz DA, Steele AD, Walson JL, Sanders JW, Mokdad AH, Murray CJL, Hay SI, Reiner RC, Jr. 2018. Morbidity and mortality due to shigella and enterotoxigenic Escherichia coli diarrhoea: the Global Burden of Disease Study 1990-2016. Lancet Infect Dis 18:1229-1240.

3. The HC, Thanh DP, Holt KE, Thomson NR, Baker S. 2016. The genomic signatures of Shigella evolution, adaptation and geographical spread. Nat Rev Microbiol 14:235-50.

4. Arena ET, Campbell-Valois FX, Tinevez JY, Nigro G, Sachse M, Moya-Nilges M, Nothelfer K, Marteyn B, Shorte SL, Sansonetti PJ. 2015. Bioimage analysis of Shigella infection reveals targeting of colonic crypts. Proc Natl Acad Sci U S A 112:E3282-90.

5. Jennison AV, Verma NK. 2004. Shigella flexneri infection: pathogenesis and vaccine development. FEMS Microbiol Rev 28:43-58.

6. Shim DH, Suzuki T, Chang SY, Park SM, Sansonetti PJ, Sasakawa C, Kweon MN. 2007. New animal model of shigellosis in the Guinea pig: its usefulness for protective efficacy studies. J Immunol 178:2476-82.

7. Wassef JS, Keren DF, Mailloux JL. 1989. Role of M cells in initial antigen uptake and in ulcer formation in the rabbit intestinal loop model of shigellosis. Infect Immun 57:85863.

8. Corr SC, Gahan CC, Hill C. 2008. M-cells: origin, morphology and role in mucosal immunity and microbial pathogenesis. FEMS Immunol Med Microbiol 52:2-12.

9. Rout WR, Formal SB, Giannella RA, Dammin GJ. 1975. Pathophysiology of Shigella diarrhea in the rhesus monkey: intestinal transport, morphological, and bacteriological studies. Gastroenterology 68:270-8.

10. Butler T, Speelman P, Kabir I, Banwell J. 1986. Colonic dysfunction during shigellosis. J Infect Dis 154:817-24.

11. Koestler BJ, Ward CM, Fisher CR, Rajan A, Maresso AW, Payne SM. 2019. Human Intestinal Enteroids as a Model System of Shigella Pathogenesis. Infect Immun 87.

12. Begley M, Gahan CG, Hill C. 2005. The interaction between bacteria and bile. FEMS Microbiol Rev 29:625-51.

13. Sistrunk JR, Nickerson KP, Chanin RB, Rasko DA, Faherty CS. 2016. Survival of the Fittest: How Bacterial Pathogens Utilize Bile To Enhance Infection. Clin Microbiol Rev 29:819-36.

14. Pope LM, Reed KE, Payne SM. 1995. Increased protein secretion and adherence to HeLa cells by Shigella spp. following growth in the presence of bile salts. Infect Immun 63:3642-8.

15. Nickerson KP, Chanin RB, Sistrunk JR, Rasko DA, Fink PJ, Barry EM, Nataro JP, Faherty CS. 2017. Analysis of Shigella flexneri Resistance, Biofilm Formation, and Transcriptional Profile in Response to Bile Salts. Infect Immun 85.

16. Hung DT, Zhu J, Sturtevant D, Mekalanos JJ. 2006. Bile acids stimulate biofilm formation in Vibrio cholerae. Mol Microbiol 59:193-201. 
17. Ridlon JM, Kang DJ, Hylemon PB. 2006. Bile salt biotransformations by human intestinal bacteria. J Lipid Res 47:241-59.

18. Evans DF, Pye G, Bramley R, Clark AG, Dyson TJ, Hardcastle JD. 1988. Measurement of gastrointestinal pH profiles in normal ambulant human subjects. Gut 29:1035-41.

19. Koziolek M, Grimm M, Becker D, Iordanov V, Zou H, Shimizu J, Wanke C, Garbacz G, Weitschies W. 2015. Investigation of pH and Temperature Profiles in the GI Tract of Fasted Human Subjects Using the Intellicap((R)) System. J Pharm Sci 104:2855-63.

20. Abuaita BH, Withey JH. 2009. Bicarbonate Induces Vibrio cholerae virulence gene expression by enhancing ToxT activity. Infect Immun 77:4111-20.

21. Koestler BJ, Waters CM. 2014. Bile acids and bicarbonate inversely regulate intracellular cyclic di-GMP in Vibrio cholerae. Infect Immun 82:3002-14.

22. Hubel KA. 1968. The ins and outs of bicarbonate in the alimentary tract. Gastroenterology 54:647-51.

23. He J, Hwang G, Liu Y, Gao L, Kilpatrick-Liverman L, Santarpia P, Zhou X, Koo H. 2016. IArginine Modifies the Exopolysaccharide Matrix and Thwarts Streptococcus mutans Outgrowth within Mixed-Species Oral Biofilms. J Bacteriol 198:2651-61.

24. Hoiby N, Ciofu O, Bjarnsholt T. 2010. Pseudomonas aeruginosa biofilms in cystic fibrosis. Future Microbiol 5:1663-74.

25. Campbell-Valois FX, Schnupf P, Nigro G, Sachse M, Sansonetti PJ, Parsot C. 2014. A fluorescent reporter reveals on/off regulation of the Shigella type III secretion apparatus during entry and cell-to-cell spread. Cell Host Microbe 15:177-89.

26. Lindsay B, Ochieng JB, Ikumapayi UN, Toure A, Ahmed D, Li S, Panchalingam S, Levine MM, Kotloff K, Rasko DA, Morris CR, Juma J, Fields BS, Dione M, Malle D, Becker SM, Houpt ER, Nataro JP, Sommerfelt H, Pop M, Oundo J, Antonio M, Hossain A, Tamboura B, Stine OC. 2013. Quantitative PCR for detection of Shigella improves ascertainment of Shigella burden in children with moderate-to-severe diarrhea in low-income countries. J Clin Microbiol 51:1740-6.

27. Williams PCM, Berkley JA. 2018. Guidelines for the treatment of dysentery (shigellosis): a systematic review of the evidence. Paediatr Int Child Health 38:S50-S65.

28. Puzari M, Sharma M, Chetia P. 2018. Emergence of antibiotic resistant Shigella species: A matter of concern. J Infect Public Health 11:451-454.

29. Wolfe AJ, Millikan DS, Campbell JM, Visick KL. 2004. Vibrio fischeri sigma54 controls motility, biofilm formation, luminescence, and colonization. Appl Environ Microbiol 70:2520-4.

30. Singh P, Brooks JF, 2nd, Ray VA, Mandel MJ, Visick KL. 2015. CysK Plays a Role in Biofilm Formation and Colonization by Vibrio fischeri. Appl Environ Microbiol 81:5223-34.

31. Musken M, Di Fiore S, Dotsch A, Fischer R, Haussler S. 2010. Genetic determinants of Pseudomonas aeruginosa biofilm establishment. Microbiology 156:431-41.

32. Tomkovich S, Dejea CM, Winglee K, Drewes JL, Chung L, Housseau F, Pope JL, Gauthier J, Sun X, Muhlbauer M, Liu X, Fathi P, Anders RA, Besharati S, Perez-Chanona E, Yang $Y$, Ding H, Wu X, Wu S, White JR, Gharaibeh RZ, Fodor AA, Wang H, Pardoll DM, Jobin C, Sears CL. 2019. Human colon mucosal biofilms from healthy or colon cancer hosts are carcinogenic. J Clin Invest 130:1699-1712. 
33. McKenney PT, Yan J, Vaubourgeix J, Becattini S, Lampen N, Motzer A, Larson PJ, Dannaoui D, Fujisawa S, Xavier JB, Pamer EG. 2019. Intestinal Bile Acids Induce a Morphotype Switch in Vancomycin-Resistant Enterococcus that Facilitates Intestinal Colonization. Cell Host Microbe 25:695-705 e5. 
A

Shigella Culture

0

Planktonic Bacteria

Biofilm

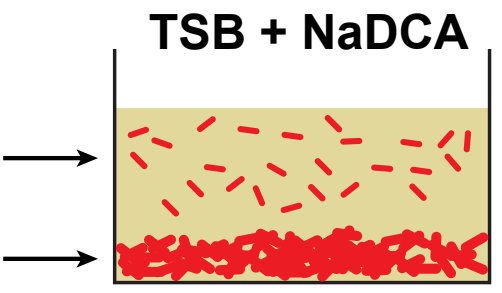

$37^{\circ} \mathrm{C} 24 \mathrm{~h}$
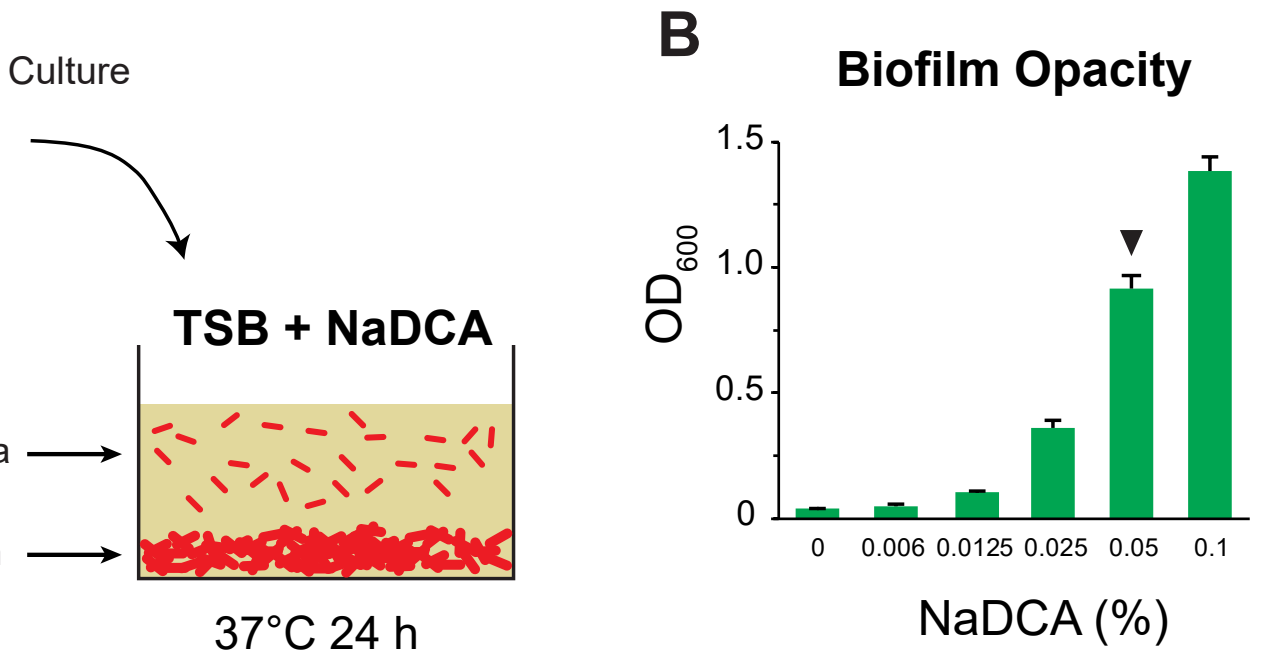

0000000 0000000 ○०००0000

OD 600 of biofilm opacity

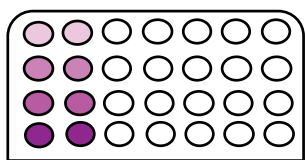

0000000 0000000 ○०0 00000

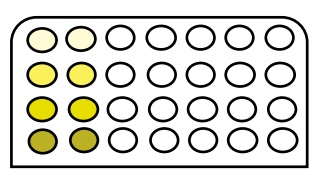

$\mathrm{OD}_{600}$ of planktonic bacteria

Culture shigella

Incubate with $0.05 \% \mathrm{NaDCA}$ under various $\mathrm{pH}$ conditions overnight

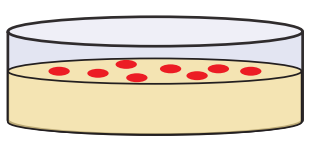

$\mathrm{CFU} / \mathrm{mL}$ of planktonic bacteria 
A

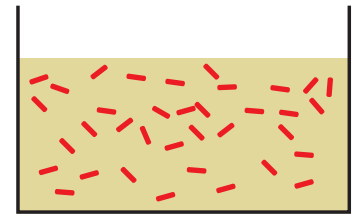

$+0.05 \% \mathrm{NaDCA}$

+ increased $\mathrm{NaOH}$

$37^{\circ} \mathrm{C} 24 \mathrm{~h}$

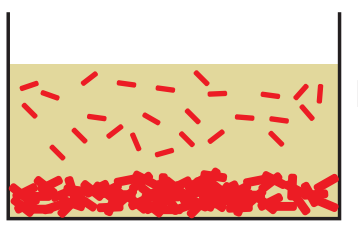

Planktonic Bacteria (Opacity, CFU)

Biofilm (Opacity, MTT)

TSB

B

\begin{tabular}{|cc|}
\hline $\mathbf{N a O H}(\mathbf{m M})$ & $\mathbf{p H}^{*}$ \\
\hline 0 & 6.9 \\
\hline 2.5 & 7.2 \\
\hline 5 & 7.4 \\
\hline 10 & 7.9 \\
\hline 20 & 8.9 \\
\hline
\end{tabular}

${ }^{*}$ measured within TSB $+0.05 \% \mathrm{NaDCA}$

E

\section{NaDCA-induced Biofilm}

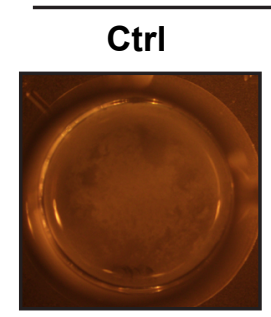

C

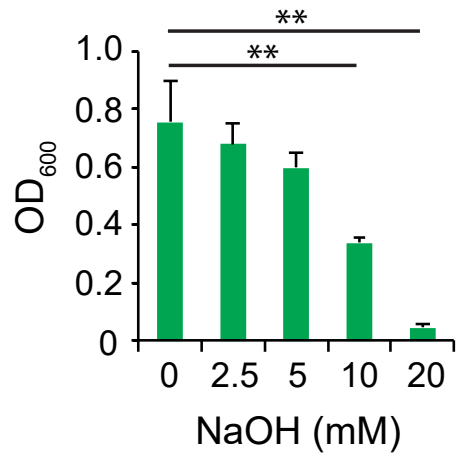

Biofilm MTT Assay

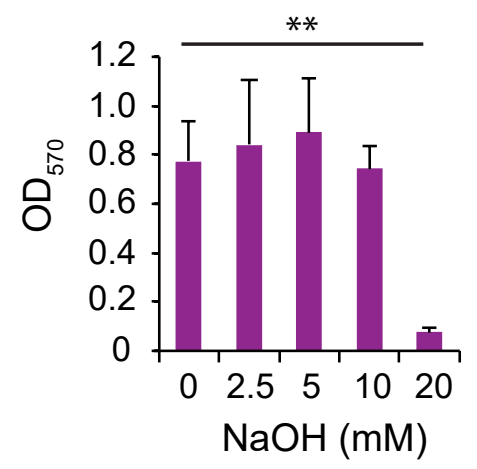

F

Planktonic Bacteria

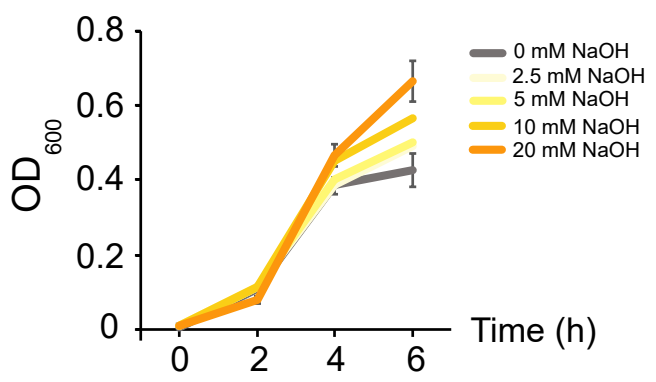

$\mathbf{G}$

Biofilm MTT Assay

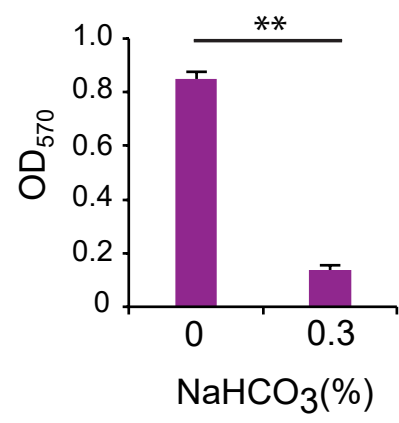

H

Planktonic Bacteria

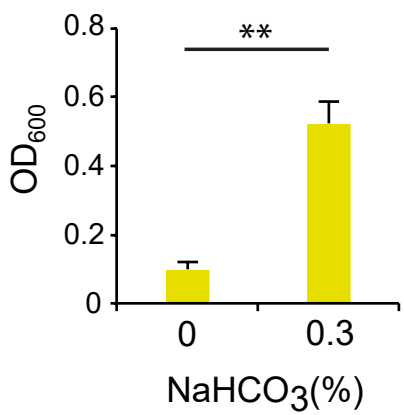

Biofilm MTT Assay

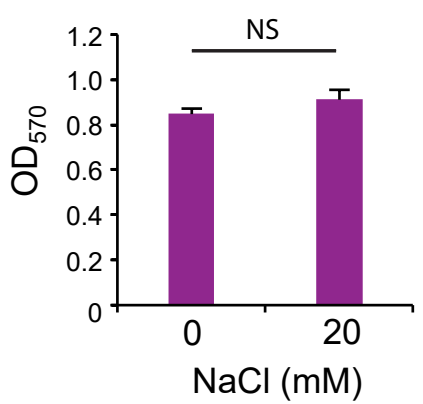


Figure 3

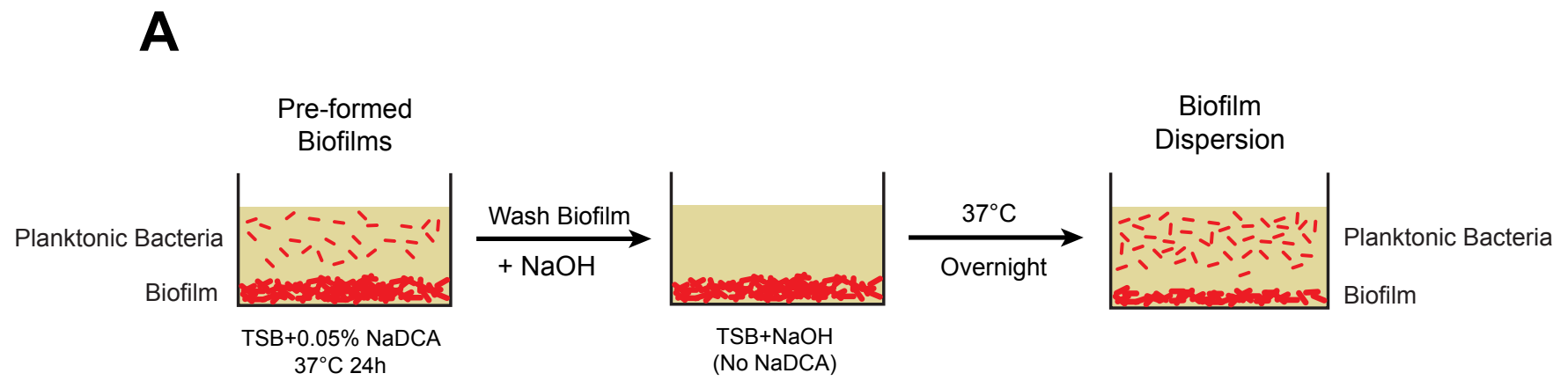

B

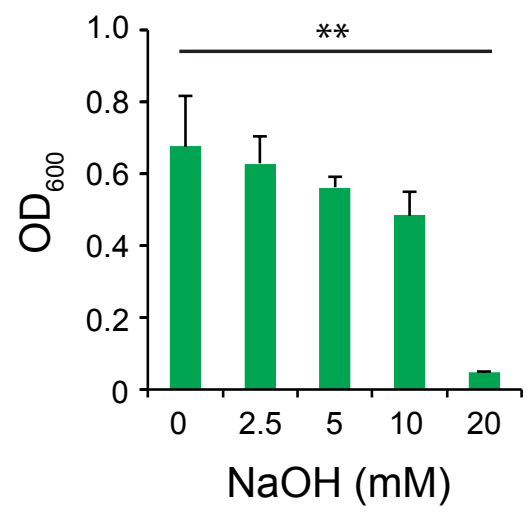

C Biofilm MTt Assay

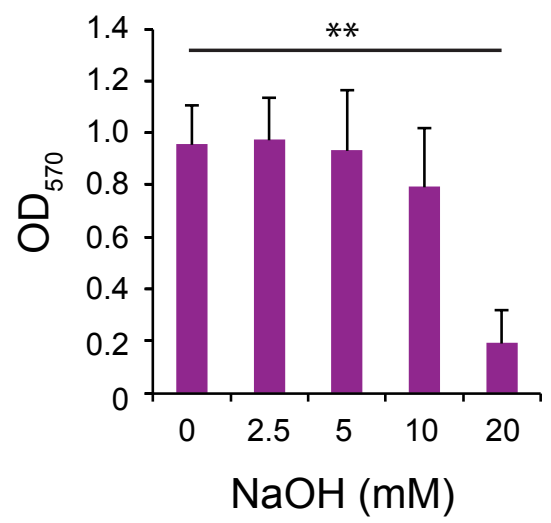


bioRxiv preprint doi: https://doi.org/10.1101/2020.10.16.336651; this version posted October 16, 2020. The copyright holder for this preprint

Figure 4

A
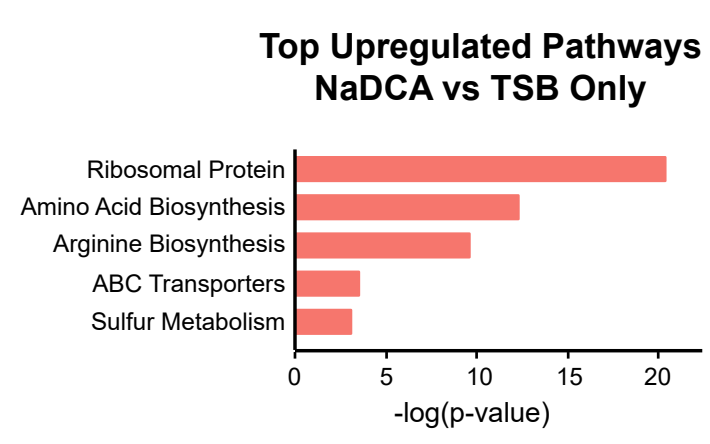

C

TSB Only $\mathrm{NaDCA} \quad \mathrm{NaDCA}+\mathrm{NaOH}$

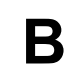

Top Downregulated Pathways $\mathrm{NaDCA}+\mathrm{NaOH}$ vs $\mathrm{NaDCA}$
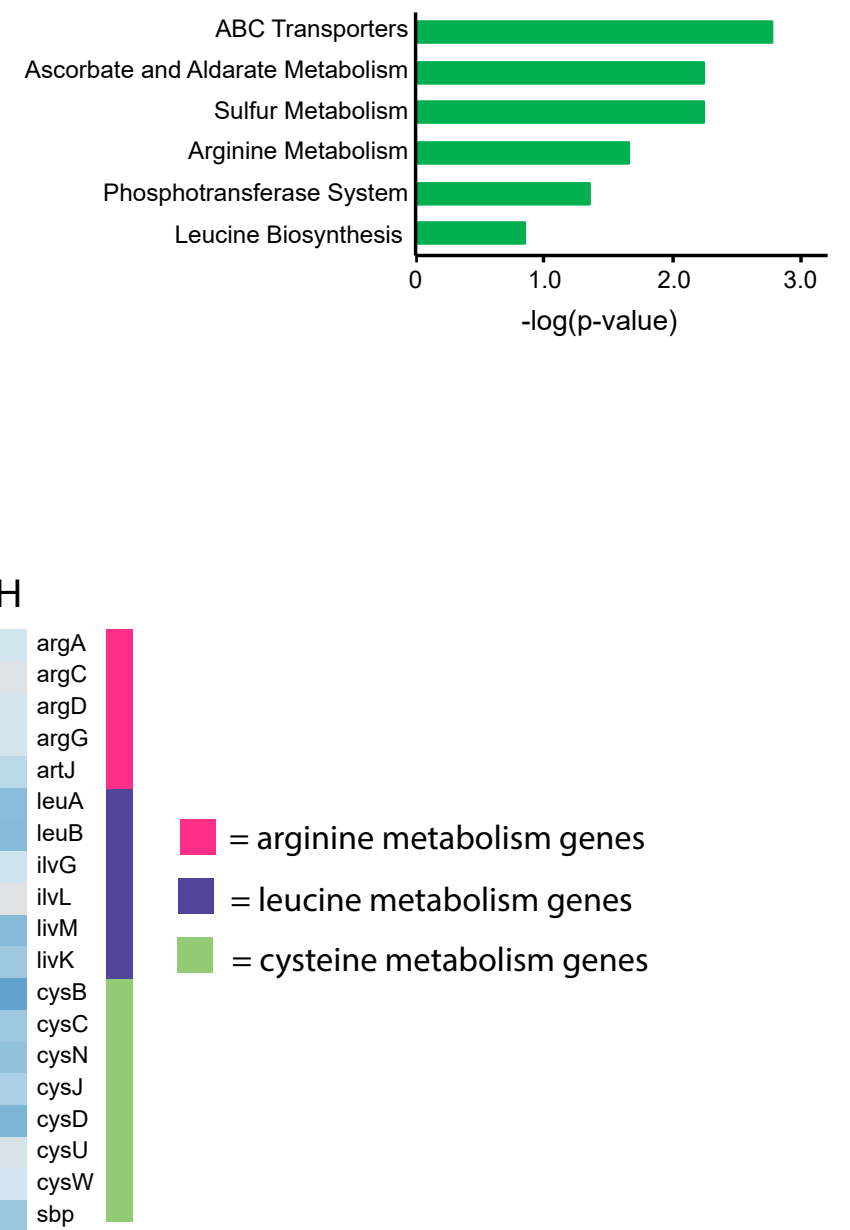

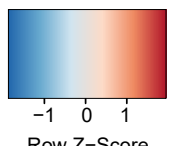


Figure 5

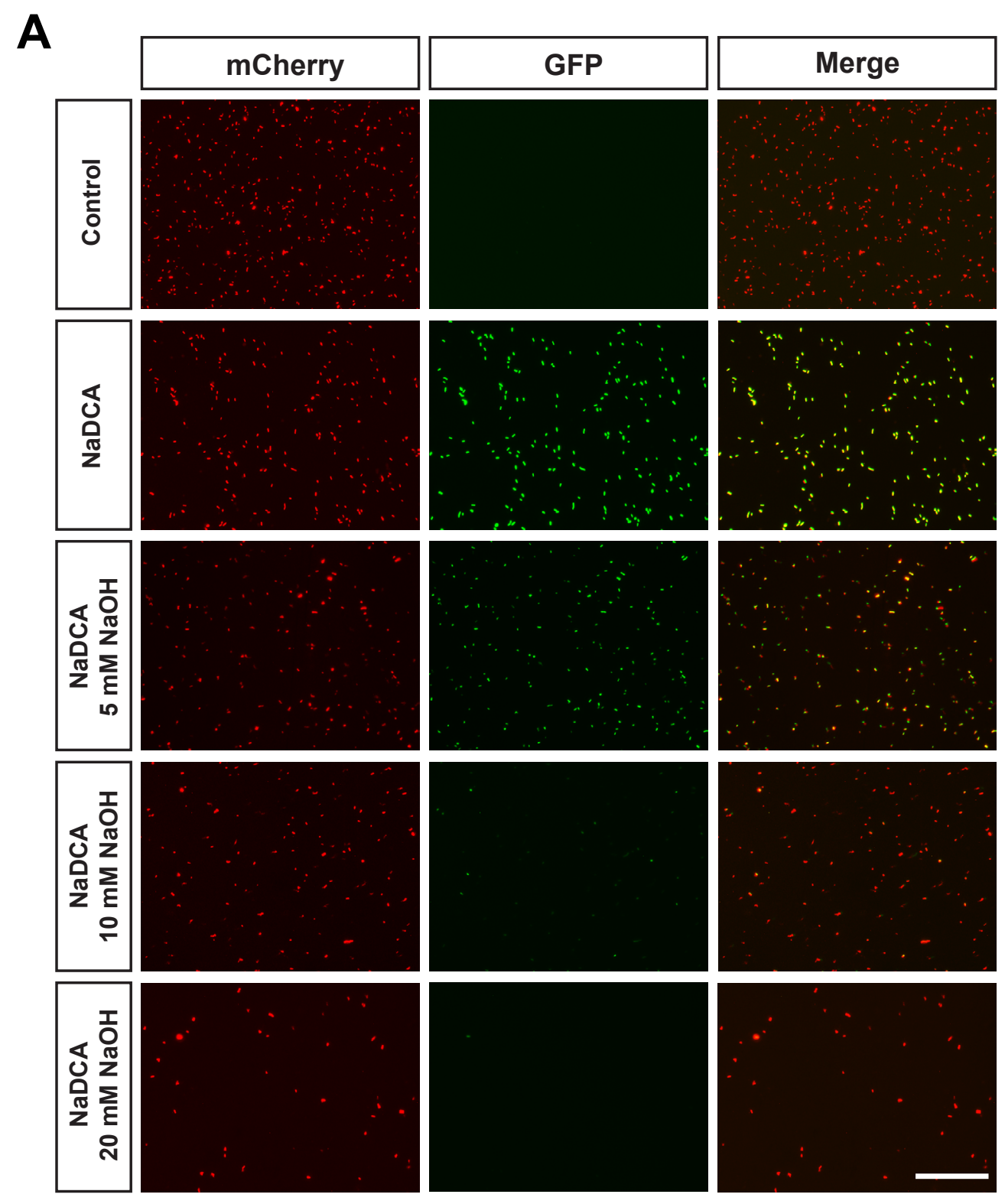

B

Percentage of GFP+ Cells

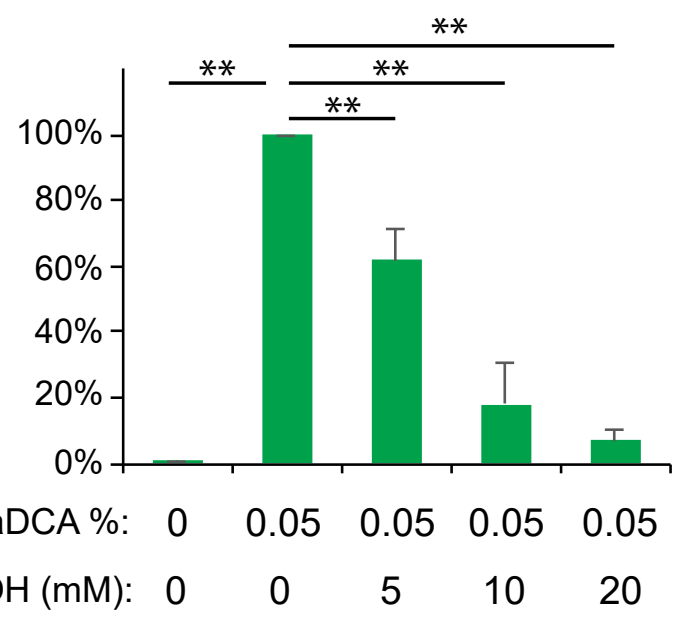


A Planktonic Bacteria B $(\mathrm{NaOH})$

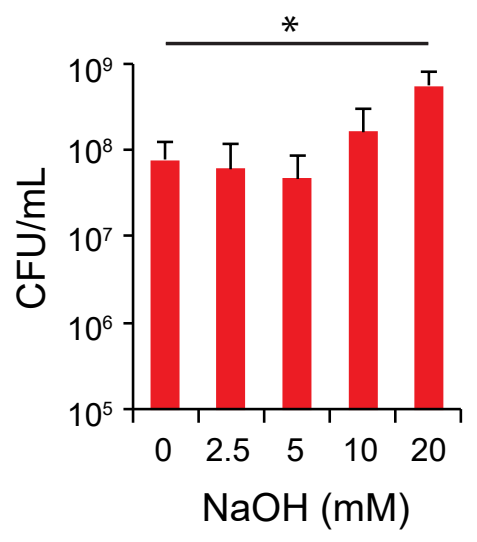

D Biofilm MTt Assay ( $\mathrm{HCl})$

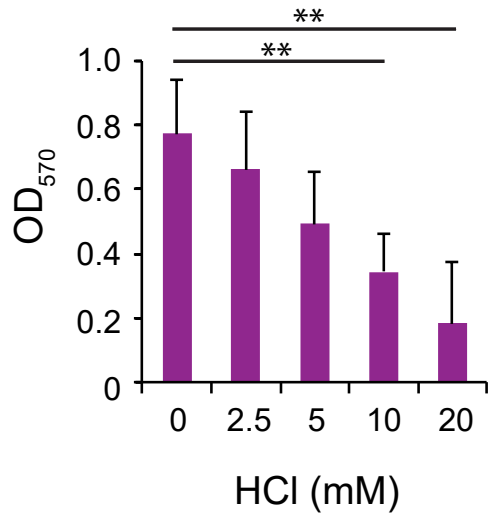

E Planktonic Bacteria ( $\mathrm{HCl})$

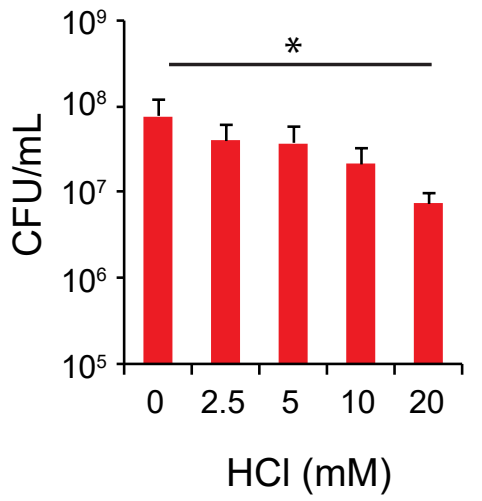

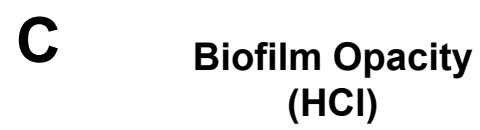

\begin{tabular}{|cc|}
\hline $\mathbf{H C l}(\mathbf{m M})$ & $\mathbf{p H}^{*}$ \\
\hline 0 & 6.9 \\
\hline 2.5 & 6.8 \\
\hline 5 & 6.6 \\
\hline 10 & 6.1 \\
\hline 20 & 4.9 \\
\hline
\end{tabular}

${ }^{*}$ measured within TSB $+0.05 \%$ NaDCA

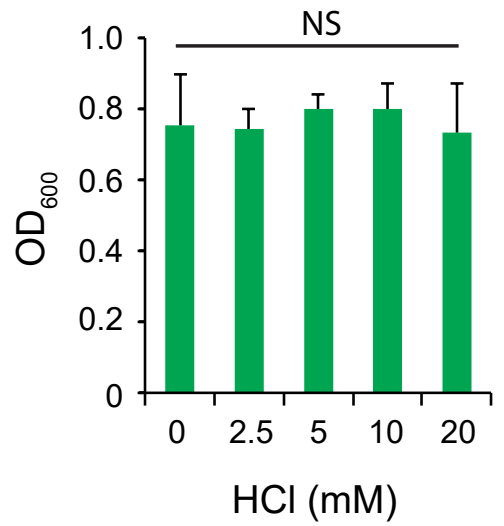

F

Planktonic Bacteria

( $\mathrm{HCl})$

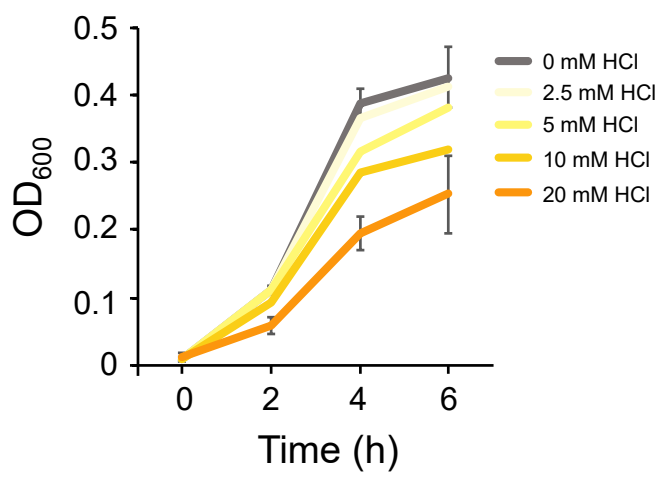


Figure S2

A

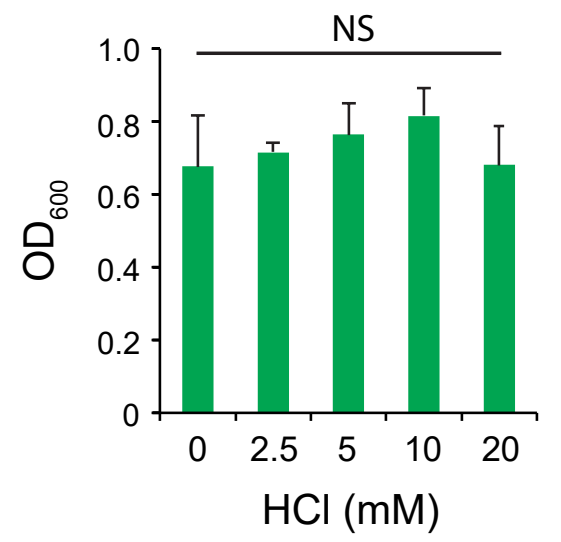

B Biofilm MTT Assay

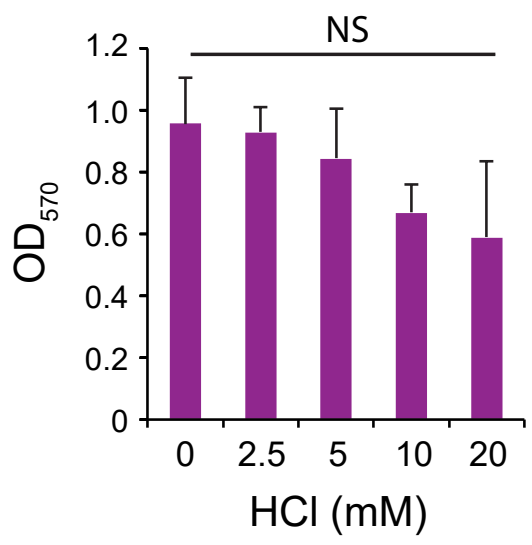




\section{Figure S3}

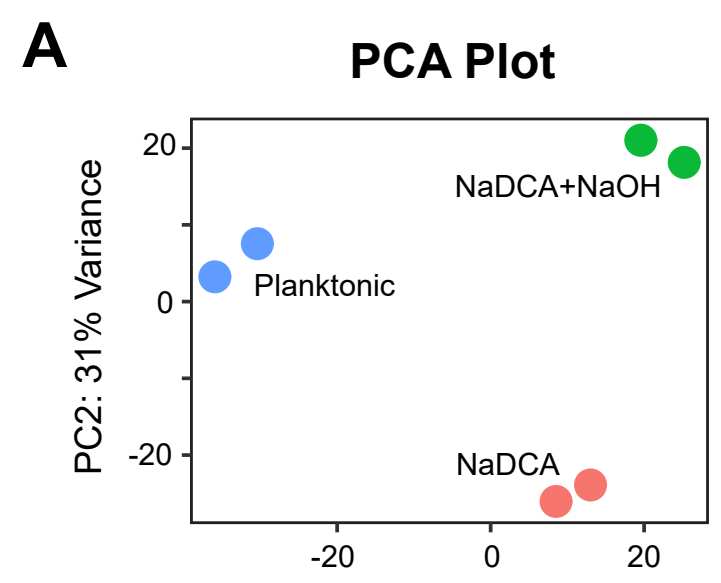

PC1: $50 \%$ Variance

B $\quad$ QPCR Validation of Targets in Amino Acid Metabolism

$\arg D(\exp 1)$

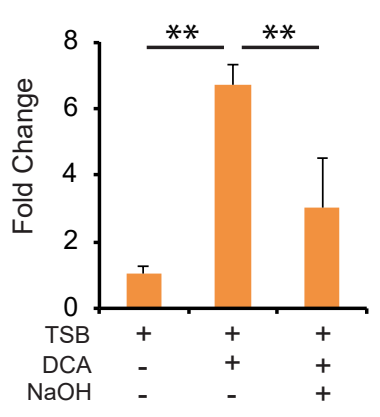

$\arg A(\exp 1)$

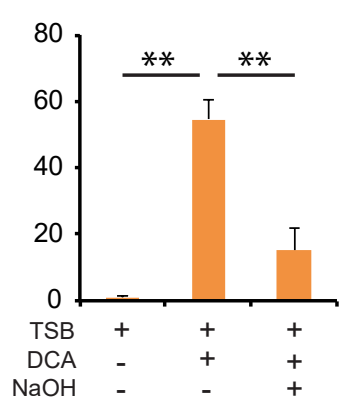

$\operatorname{argD}(\exp 2)$

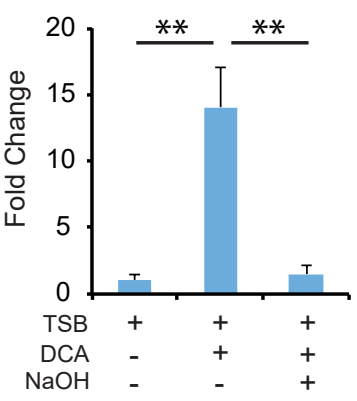

$\operatorname{argA}(\exp 2)$

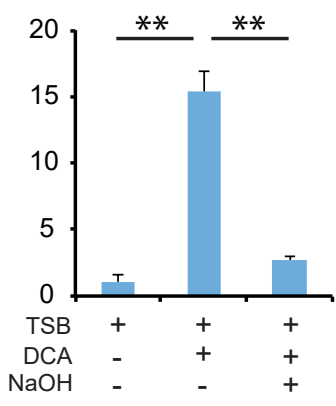

$\operatorname{argC}(\exp 1)$

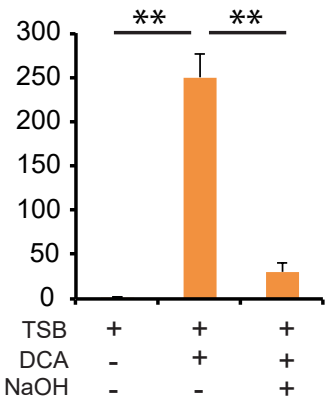

cysW (exp1)

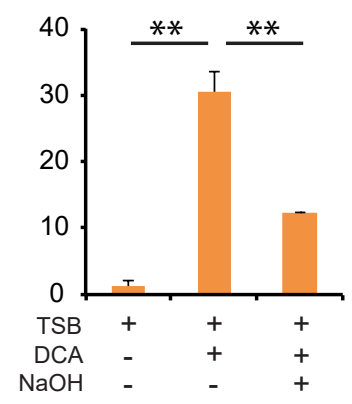

cysJ (exp 1)

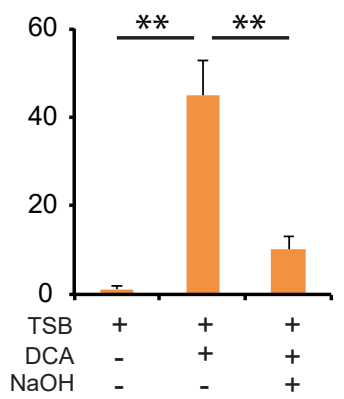

cysW (exp 2)

cysJ (exp 2)
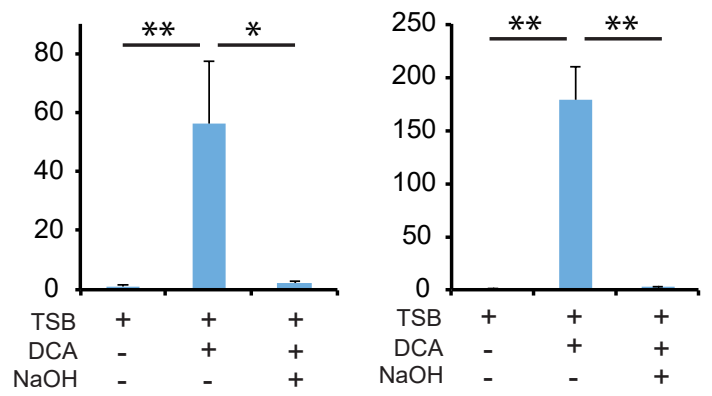